\title{
Exclusive and any breast-feeding rates of Pacific infants in Auckland: data from the Pacific Islands Families First Two Years of Life study
}

\author{
Philip J Schluter ${ }^{1, *}$, Sarnia Carter $^{1}$ and Teuila Percival ${ }^{2}$ \\ ${ }^{1}$ Faculty of Health and Environmental Sciences, Auckland University of Technology, Private Bag 92006, \\ Auckland 1020, New Zealand: ${ }^{2}$ Kidz First Children's Hospital and Community Services, Auckland, New Zealand
}

Submitted 28 June 2005: Accepted 14 December 2005

\begin{abstract}
Objectives: To present current breast-feeding rates for Pacific infants resident in New Zealand. Reasons for the introduction of complementary liquid foods were also explored.

Design: A longitudinal study using hospital discharge summary records and maternal home interviews undertaken at 6 weeks, 12 and 24 months postpartum. Turnbull's non-parametric survival analysis was used to model exclusive breast-feeding rates. Setting: Auckland, New Zealand.

Results: The cohort comprised 1376 infants at 6 weeks, 1223 infants at 12 months and 1142 infants at 24 months. Exclusive breast-feeding rates at hospital discharge, 6 weeks, 3 and 6 months postpartum were 84\% (95\% confidence interval (CI): 80-88\%), 49\% (95\% CI: 43-55\%), 37\% (95\% CI: 32-42\%) and 9\% (95\% CI: 7-11\%), respectively. Significant ethnic difference existed, with Samoan mothers having higher exclusive breast-feeding rates than Tongan mothers $(P=0.002)$. The percentage of infants receiving any breast milk at hospital discharge, 6 weeks, 12 and 24 months was 96\% (95\% CI: 94-97\%), 95\% (95\% CI: 94-96\%), 31\% (95\% CI: 28-34\%) and 15\% (95\% CI: 13-17\%), respectively. Again ethnic differences emerged. Common reasons cited for discontinuation of exclusive breast-feeding included uncertainty of breast milk supply (56\%), problems with breasts (30\%) and difficulties breast-feeding in work or educational environments (26\%). However, 691 (50\%) mothers sought no advice about their breast-feeding concerns within the first six weeks of life.

Conclusions: Exclusive breast-feeding rates for Pacific infants are ethnically heterogeneous, have declined since the 1990s and fall short of the World Health Organization recommendations. The principal reasons cited for exclusive breastfeeding discontinuation echo those reported over a decade ago.
\end{abstract}

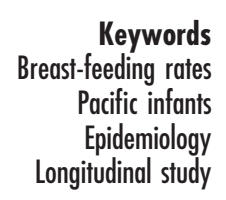

Breast-feeding confers substantial benefits to both mother and child ${ }^{1}$. Following a systematic review of current scientific evidence on the optimal duration of exclusive breast-feeding ${ }^{2}$, a recent international meeting of experts coordinated under the auspices of the World Health Organization (WHO) continued to recommend exclusive breast-feeding for the first six months, with introduction of complementary foods and continued breast-feeding thereafter $^{3}$. Many other international organisations concur with this recommendation ${ }^{1}$. However, the WHO Global Data Bank on Breastfeeding and Complementary Feeding, which presently covers 94 countries and 65\% of the world's infant population, estimates that only $35 \%$ of infants are exclusively breast-fed between 0 and 4 months of age ${ }^{4}$. Rates between countries vary widely, from extremely low (e.g. Nigeria: 2\% in 1992, Central African Republic: 4\% in 1995) in the African Region to 68\% in 1995 for Egypt and 61\% in 1993 for Sweden ${ }^{4}$.
Within the New Zealand context, Pacific peoples are one of the fastest growing subpopulations 5 , now estimated to exceed 284000 people comprising 6.8\% of the total population ${ }^{6}$, and Auckland is their preferred region of domicile ${ }^{7}$. However, Pacific people are overrepresented in multiple adverse health and social statistics $^{6,7}$. Results from the Plunket National Child Study, conducted on a birth cohort of 4286 infants born during 1990-91, demonstrated that overall exclusive breast-feeding rates at hospital discharge, 6 weeks, 3 months and 6 months postpartum were 93.8\%, 68.4\%, $47.6 \%$ and $2.5 \%$, respectively ${ }^{8}$. Exclusive breast-feeding rates for the Pacific sub-sample at hospital discharge, 6 weeks, 3 months and 6 months postpartum were comparable at 93\%, 70\%, 52\% and $2 \%$, respectively ${ }^{8}$. However, in a birth cohort study of Pacific infants resident in New Zealand we recently identified that breast-feeding rates at hospital discharge and 6 weeks were below those 
derived from the Plunket National Child Study and that internationally recommended ${ }^{1,3,8,9}$. The present paper extends these findings to report current exclusive and any breast-feeding rates for the Pacific cohort over infants' first two years of life.

Previous New Zealand studies have consistently found that the most common reason for the supplementation of breast milk or cessation of breast-feeding was a perceived inadequate milk supply ${ }^{8,10-13}$. Essex and colleagues concluded that the decline in breast-feeding rates with length of time postpartum could be reduced with education of mothers and health professionals about the management of breast-feeding problems, and increased support from employers or educational facilities ${ }^{8}$. Using data across the first two years of life, a second aim of this paper is to report the reasons for the introduction of complementary liquid or solid foods. From this we can gauge whether mother's perceptions of barriers to breastfeeding have changed from those observed over a decade ago.

\section{Methods}

Data were collected as part of the Pacific Islands Families: First Two Years of Life (PIF) study. This study follows a cohort of Pacific Islands' infants born at Middlemore Hospital between 15 March and 17 December 2000. The rationale for selecting Middlemore Hospital was that approximately two-thirds of Pacific communities resident in New Zealand live within the Auckland region ${ }^{14}$ and this hospital has the largest number of Pacific births in the country, with all major Pacific ethnic groups represented. All potential participants were selected from births where at least one parent was identified as being of Pacific Island ethnicity and a New Zealand permanent resident. Participants were identified through the Birthing Unit, in conjunction with the Pacific Islands Cultural Resource Unit. Information about the study was provided and consent was sought to make a home visit.

Approximately six weeks after the infant's birth, female interviewers of Pacific Island ethnicity who were fluent in English and a Pacific Islands language visited the mother in her home. Once eligibility was confirmed and informed consent obtained, mothers participated in a one hour interview concerning family functioning and the health and development of the child. This interview was conducted in the preferred language of the mother. With consent, home visits were repeated at approximately 12 months and 24 months postpartum. Detailed information about the cohort and procedures is described elsewhere ${ }^{15}$.

\section{Measures of breast-feeding}

Breast-feeding at hospital discharge was obtained from Middlemore Hospital's hospital discharge summary records for each mother. At the 6-week interview, mothers were asked how they fed their baby for the first six weeks.
Problems with breast-feeding, reasons for the introduction of complementary liquid or solid foods, and whether advice was sought for infant feeding and from whom, were also elicited. At the 12-month interview, the current method of feeding was elicited from options that included: exclusive breast-feeding (breast milk only); combining breast-feeding with other feeding (formula or solid food); not breast-feeding, feeding only formula or other milk; not breast-feeding, combining formula/other milk and solid food; and other (describe). Participants were also asked about the feeding of other liquids in the last seven days including: water, juices, coconut derivatives, soft drinks, tea or coffee. The age of introduction of complementary liquid foods was elicited, as was the age when complementary solid foods were introduced where applicable. Reasons why complementary liquid foods were introduced were also asked. For the 24-month interview mothers were asked whether their child had breast milk at any time in the past seven days.

Infants were defined as exclusively breast-fed if they were described at discharge from hospital as being breastfed and they continued to receive only breast milk until the age in question. Infants were deemed not to be exclusively breast-fed if any complementary solid or liquid foods (except minimal amounts of water or prescribed medicine) were consumed by the infant before the age in question or if there was any inconsistency between the elicited answers.

\section{Statistical analysis}

All infants who were singletons or first-borns from multiple births were included within the analyses. Crosstabulations of feeding variables collected between interview waves were conducted to find inconsistencies in response. If mothers claimed to be exclusively breastfeeding at one interview but not exclusively breast-feeding at a previous interview, then the exclusive breast-feeding response was modified to non-exclusive breast-feeding. A similar strategy was used for the any breast-feeding variables. Also, the ages that infants were introduced complementary liquid foods or solids were compared with the breast-feeding variables. If mothers claimed to be exclusively breast-feeding at a give point in time yet provided information in another question that the infant had received complementary liquid foods or solids before that time, then the exclusive breast-feeding response was modified to that of nonexclusive breast-feeding.

The likelihood that infants were exclusively breastfeeding over age postpartum was modelled using Turnbull's non-parametric survival analysis ${ }^{16}$. This technique allows both interval and right censoring of the event of interest over time. In these analyses the event was taken to be the feeding of an infant complementary liquid or solid foods and the time component was deemed to be the age at which this 
event occurred. In instances where the time component was missing yet information was available from the interview or subsequent interviews that exclusive breastfeeding had discontinued, then time was assigned as the interval beginning at the last known age where the infant was exclusively breast-fed and ending at the earliest age at which it was known that the infant was no longer exclusively breast-fed. If the age at which the infant was no longer exclusively breast-fed was unavailable, then observations were treated as being right censored and time was equated to that last known age of exclusive breast-feeding. Hypothesis tests between Turnbull's nonparametric survival curves were based on a modified log rank test and calculated using the PROC IML algorithm originated by $\mathrm{So}^{17}$ and adapted by Lindsey and Ryan ${ }^{16}$.

Rates of any breast-feeding were determined crosssectionally at each interview time point and confidence intervals were calculated using an exact binomial method. Fisher's exact test was used to compare rates across ethnic groups and assess whether there was differential loss to follow-up. SAS version $8.2^{18}$ and Stata version $8.0^{19}$ were used for all computations and a level of $P<0.05$ was used to define statistical significance.

\section{Etbics}

Ethical approval was obtained from the Auckland Branch of the National Ethics Committee, the Royal New Zealand Plunket Society, and the South Auckland Health Clinical Board.

\section{Results}

Overall, 1708 mothers were identified, 1657 were invited to participate, 1590 (96\%) consented to a home visit and, of these, 1477 (93\%) were eligible for the PIF study. Of those eligible, 1376 (93\%) participated at the 6-week interview, 1224 (83\%) participated at the 12-month interview and 1144 (77\%) participated at the 24-month interview. At the 6-week interview, mothers' self-identified major ethnic affiliations included 650 (47\%) Samoan, 289 (21\%) Tongan, 232 (17\%) Cook Island Maori, 59 (4\%) Niuean, 47 (3\%) other Pacific (including mothers who identified equally with two or more ethnic groups) and 99 (7\%) non-Pacific (infants with non-Pacific mothers and Pacific fathers). Mothers' mean age was 27.9 years (standard deviation 6.2 years), 1107 (80\%) were married or in de facto relationships, 377 (27\%) gained postsecondary school qualifications, 343 (25\%) admitted to current cigarette smoking, and 56 (4\%) were in full-time and 28 (2\%) were in part-time employment (see Table 1).

Of the 1376 selected infants, 662 (48\%) were female, the mean infant birth weight was $3583 \mathrm{~g}$ (standard deviation $616 \mathrm{~g}$ ) with 65 (5\%) recording a birth weight under $2500 \mathrm{~g}$, 107 (8\%) infants were born pre-term with gestational age $<37$ weeks, and 37 (3\%) were discharged from hospital at a different time to their mother (see Table 1).
At the 6-week interview, the 1376 infants had a median age of 7 weeks and $95 \%$ of interviews were completed with infants aged between 5 and 14 weeks. Hospital discharge information on infant feeding was available for 1260 (92\%) of these infants. The 12-month interview was conducted on 1223 infants; their median age was 12.3 months and 95\% of interviews were completed with infants aged between 11.9 and 17.1 months. Finally, the 24-month interview was conducted on 1142 infants; their median age was 24.1 months and $95 \%$ of interviews were completed with infants aged between 23.8 and 26.6 months.

\section{Exclusive breast-feeding}

At the 6-week interview, 731 (53\%) mothers declared that they were exclusively breast-feeding yet of these 26 (2\%) were complementary feeding or fully formula-feeding at hospital discharge and a further 22 (2\%) declared that they had introduced complementary liquid foods before 6 weeks. The status of these 48 responses was changed to non-exclusive breast-feeding. At hospital discharge, the exclusive breast-feeding rate was estimated as 84\% (95\% confidence interval (CI): 80-88\%), declining to $49 \%(95 \% \mathrm{CI}: 43-55 \%)$ at 6 weeks postpartum, $37 \%$ (95\% CI: $32-42 \%)$ at 3 months postpartum and 9\% (95\% CI: $7-11 \%)$ at 6 months postpartum. No mother reported exclusively breastfeeding at the 12-month or 24-month interview. Figure 1 depicts the overall Turnbull non-parametric survival curve of exclusive breast-feeding for this sample.

There was a significant difference in the survival curves for exclusive breast-feeding between the self-identified major ethnic affiliations $(P=0.04)$, see Fig. 2 . Of the three largest ethnic groups, Samoan mothers had exclusive breast-feeding rates that were significantly higher than Tongan mothers $(P=0.002)$. However, the exclusive breast-feeding rates of the Cook Island Maori mothers were not significantly different from either the Samoan mothers $(P=0.10)$ or the Tongan mothers $(P=0.28)$, see Fig. 2.

Exclusive breast-feeding rates were also significantly associated with maternal smoking $(P<0.001)$, maternal employment $(P<0.001)$, having a low-birth-weight baby $(P=0.001)$ and whether the mother and baby were discharged from hospital together $(P=0.008)$, see Table 1.

At the 6-week interview, 214 (16\%) infants had not been visited at home by a Plunket nurse (in New Zealand, child health nurses are called Plunket nurses and are employed by the Royal New Zealand Plunket Society ${ }^{20}$ ). No statistically significant difference existed between the Turnbull non-parametric survival curves for exclusive breast-feeding between those with and without Plunket visits $(P=0.40)$. The exclusive breast-feeding rate at hospital discharge, 6 weeks, 3 months and 6 months was estimated at $84 \%, 50 \%, 38 \%$ and $10 \%$, respectively, for 
Table 1 Frequencies, percentages and exclusive breast-feeding rate estimates derived from the Turnbull non-parametric survival analysis at 6 weeks, 3 months and 6 months postpartum partitioned by maternal and infant characteristics measured at 6 weeks

\begin{tabular}{|c|c|c|c|c|c|}
\hline & \multirow[b]{2}{*}{$n(\%)$} & \multicolumn{3}{|c|}{ Exclusive breast-feeding rate (\%) } & \multirow[b]{2}{*}{$P$-value } \\
\hline & & 6 weeks & 3 months & 6 months & \\
\hline \multicolumn{5}{|l|}{ Mother's age (years)* } & 0.27 \\
\hline$<25$ & 465 (34) & 51 & 37 & 10 & \\
\hline $25-35$ & $430(31)$ & 51 & 37 & 8 & \\
\hline$>35$ & $480(35)$ & 46 & 37 & 9 & \\
\hline \multicolumn{5}{|l|}{ Mother's parity $\dagger$} & 0.22 \\
\hline 1 & $374(28)$ & 51 & 35 & 10 & \\
\hline $2-4$ & $768(57)$ & 50 & 39 & 8 & \\
\hline$\geq 5$ & 215 (16) & 41 & 34 & 10 & \\
\hline \multicolumn{5}{|l|}{ Mother’s ethnicity $\ddagger$} & 0.04 \\
\hline Samoan & $650(47)$ & 55 & 43 & 10 & \\
\hline Tongan & $289(21)$ & 40 & 25 & 7 & \\
\hline Cook Island Maori & $232(17)$ & 46 & 34 & 10 & \\
\hline Niuean & $59(4)$ & 53 & 41 & 6 & \\
\hline Other Pacific & 47 (3) & 50 & 37 & 3 & \\
\hline Non-Pacific & $99(7)$ & 46 & 33 & 5 & \\
\hline \multicolumn{5}{|l|}{ Mother's martial status } & 0.83 \\
\hline Partnered & $1107(80)$ & 49 & 37 & 8 & \\
\hline Single & $269(20)$ & 50 & 35 & 11 & \\
\hline \multicolumn{5}{|l|}{ Mother's highest level of education } & 0.59 \\
\hline Primary & 535 (39) & 49 & 36 & 7 & \\
\hline Secondary & 464 (34) & 51 & 39 & 10 & \\
\hline Post-secondary & 377 (27) & 49 & 35 & 8 & \\
\hline \multicolumn{5}{|l|}{ Mother's smoking status§ } & $<0.001$ \\
\hline Non-smoker & $1029(75)$ & 54 & 41 & 11 & \\
\hline Smoker & $343(25)$ & 36 & 26 & 3 & \\
\hline \multicolumn{5}{|l|}{ Mother's employment status } & $<0.001$ \\
\hline Not working & $1292(94)$ & 51 & 39 & 10 & \\
\hline Part-time & $28(2)$ & 18 & 11 & 4 & \\
\hline Full-time & $56(4)$ & 21 & 11 & 0 & \\
\hline \multicolumn{5}{|l|}{ Plunket visited home postpartum } & 0.40 \\
\hline Yes & $1159(84)$ & 50 & 38 & 10 & \\
\hline No & $214(16)$ & 45 & 31 & 8 & \\
\hline \multicolumn{5}{|l|}{ Infant's sex } & 0.40 \\
\hline Female & $662(48)$ & 49 & 38 & 10 & \\
\hline Male & $714(52)$ & 50 & 36 & 7 & \\
\hline \multicolumn{5}{|l|}{ Gestation (weeks)\| } & 0.31 \\
\hline$\geq 37$ & $1248(92)$ & 50 & 38 & 9 & \\
\hline$<37$ & $107(8)$ & 40 & 28 & 14 & \\
\hline \multicolumn{6}{|l|}{ Birth weight $(g) \dagger$} \\
\hline$\geq 2500$ & $1292(95)$ & 50 & 38 & 10 & 0.001 \\
\hline$<2500$ & $65(5)$ & 30 & 14 & 7 & \\
\hline \multicolumn{5}{|l|}{ Hospital discharge of mother and infants } & 0.008 \\
\hline Together & $1339(97)$ & 50 & 38 & 10 & \\
\hline Separately & $37(3)$ & 24 & 15 & 6 & \\
\hline
\end{tabular}

* One observation missing.

† Nineteen observations missing.

¥ 'Other Pacific' includes mothers identifying equally with two or more ethnic groups and 'non-Pacific' includes mothers who were eligible through the Pacific ethnicity of the father.

$\S$ Four observations missing.

I Three observations missing.

\| Twenty-one observations missing.

those with visits and $85 \%, 45 \%, 31 \%$ and $8 \%$, respectively, for those without such visits.

The reasons mothers introduced complementary liquid foods into infants' diets were many and diverse. For the 1085 mothers providing such reasons, the 10 most common appear in Table 2 .

While many of these reasons or perceptions were surmountable, 691 (50\%) sought no advice about concerns they had with infant feeding within the first six weeks of life. Of the 685 mothers who did seek advice, 594 (87\%) mothers consulted midwives, 244 (36\%) consulted other women in the family, 241 (35\%) consulted Plunket nurses, $103(15 \%)$ consulted general practitioners and 77 (11\%) consulted practice nurses about concerns they had about infant feeding.

\section{Any breast-feeding}

No inconsistencies in any breast-feeding responses were found between interviews for any mothers. The percentage of infants receiving at least some breast milk at 
696

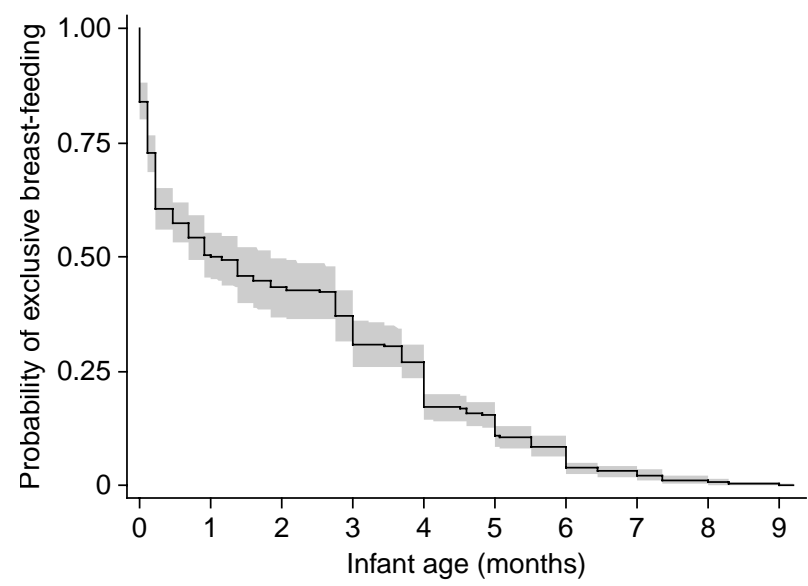

Fig. 1 Turnbull non-parametric survival curve estimates for exclusive breast-feeding and associated $95 \%$ confidence intervals (given by the shaded region)

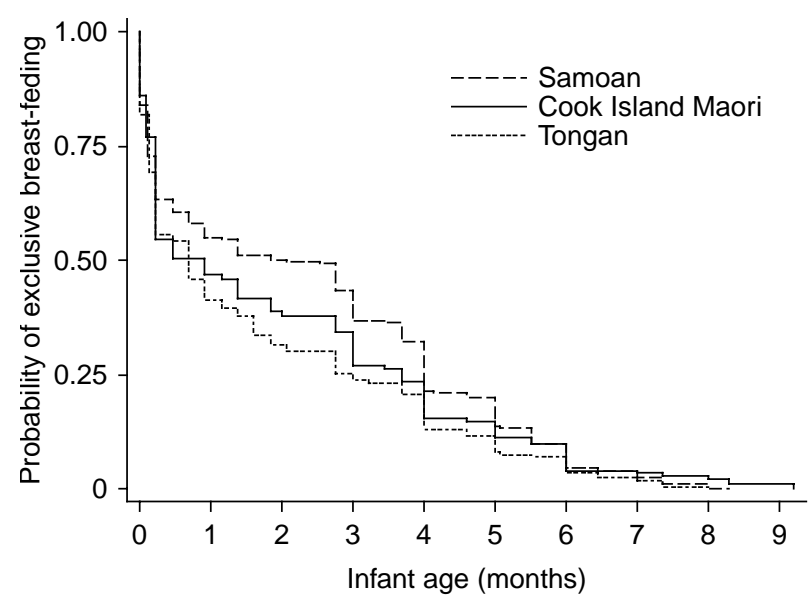

Fig. 2 Turnbull non-parametric survival curve estimates for exclusive breast-feeding for the three largest Pacific ethnicities

hospital discharge, 6 weeks, 12 months and 24 months was 96\% (95\% CI: 94-97\%), 95\% (95\% CI: 94-96\%), 31\% (95\% CI: 28-34\%) and 15\% (95\% CI: 13-17\%), respectively. There were no ethnic differences between the any
PJ Schluter et al.

breast-feeding rates at hospital discharge $(P=0.46)$ or at the 6-week interview $(P=0.48)$. However, significant ethnic differences emerged at the 12-month interview (Samoan 39\%, Cook Island Maori 25\%, Tongan 23\%, $P<0.001$ ) and at the 24-month interview (Samoan 21\%, Cook Island Maori 18\%, Tongan 14\%, $P<0.001$ ).

Of those lost to follow-up at the 12 month interview, $96 \%$ were breast-fed and $4 \%$ were formula-fed at the 6week interview, no different from those remaining in the study $(P=0.69)$. Similarly, of those lost to follow-up at the 24-month interview, 94\% were breast-fed and 6\% were formula fed at the 6-week interview, no different from those remaining in the study $(P=0.51)$.

\section{Discussion}

In this large prospective study of Pacific infants resident within the Auckland region, New Zealand, we report exclusive breast-feeding rates at hospital discharge, 6 weeks, 3 months and 6 months that have fallen by an absolute percentage difference of up to $17 \%$ on those reported over a decade earlier ${ }^{8}$. In the intervening years, data gathered by Plunket also demonstrate a decline in national rates for full breast-feeding at 6 weeks: from $75 \%$ in 1994 to $64 \%$ in 1996-97 ${ }^{21}$. More recent 2001 figures from the New Zealand Ministry of Health suggest that the national fully breast-feeding rates for Pacific infants at 5-6 weeks, 3 months and 4-6 months were 57\%, 43\% and $17 \%$, respectively ${ }^{22}$. These Ministry figures were largely static over the 5-year reporting period from 1997 to 2001. Within Auckland, the July to December 2001 Ministry figures for full breast-feeding at 6 weeks and 11-15 weeks were $55 \%$ and $41 \%$, respectively; $2 \%$ less than the national figures $^{22}$.

Our reported exclusive breast-feeding rates for Pacific infants during 2001 are lower, and some considerably lower, than the published Ministry figures; although the Ministry's use of age bracket makes direct comparison and interpretation difficult. For instance, if we consider the exclusive breast-feeding rates within our sample, we find $27 \%$ are breast-feeding at 4 months declining to $9 \%$ at 6

Table 2 The 10 most common reasons cited by mothers for introducing complementary liquid foods into infants diets. More than one reason may have been given

\begin{tabular}{llc}
\hline Rank & \multicolumn{1}{c}{ Reason for introducing complementary liquid foods } & $n(\%)$ \\
\hline 1 & Unsure about adequate milk supply from breast-feeding & $604(56)$ \\
2 & Problems with breasts (cracked nipples, infections, etc.) & $324(30)$ \\
3 & Difficulties with breast-feeding due to return to work or study & $281(26)$ \\
4 & Baby refused to feed or had sucking difficulties with breast & $260(24)$ \\
5 & Baby was unsettled & $198(18)$ \\
6 & Concern about lack of weight gain in baby & $167(15)$ \\
7 & Lack of freedom or inconvenience of breast-feeding & $144(13)$ \\
8 & Felt too tired or stressed to breast-feed & $131(12)$ \\
9 & Formula-fed so that partner could share feeding of baby & $122(11)$ \\
10 & Healthiest way to feed my baby & $92(8)$ \\
\hline
\end{tabular}


months. Thus, the quoted Ministry figure of $17 \%$ fully breast-feeding at 4-6 months has little utility, and changes in the Ministry figures may simply result from fluctuations in the infant age profile of the captured mothers. This limitation is explicitly acknowledged by the Ministry, as are the inconsistencies in breast-feeding definitions used between various information sources and the percentage of the population from whom the data are captured ${ }^{22}$.

Differences between the Ministry's figures and our estimates may also result from discrepancies between sampling-frame coverage. Specifically, the Ministry's 5-6 weeks Pacific figures are derived from an estimated 71$75 \%$ of Pacific births, while 3 months and 6 months Pacific figures cover an estimated $82-90 \%$ of Pacific births ${ }^{22}$. The PIF study used an inception cohort of all mothers giving birth to Pacific infants at Middlemore Hospital between 15 March and 17 December 2000, and there was no important differential attrition noted across a range of sociodemographic variables investigated ${ }^{15}$ or in the breast-feeding frequencies at the 6-week interview between those who dropped out and those who continued in the study. The PIF study sample thus covers an additional 25-29\% of Pacific births at the 6-week interview compared with that of the Ministry. Unfortunately, the Ministry's sampling frame for the 5-6 week estimates is not specified beyond public health, maternity and Well Child services (a service that provides screening, surveillance, education and support services to all New Zealand infants and their families which is linked to, and follows on from, the care provided by the lead maternity carer) and so the magnitude of the selection bias cannot be precisely quantified $^{23}$. However, it might be opined that those not captured within the Ministry's sampling frame are likely to have lower breast-feeding rates than those captured due to a number of factors including socio-economic ${ }^{24}$ and educational $^{25,26}$ disparity, employment and workplace support differences ${ }^{27}$, and behavioural and childcare practice variations ${ }^{26}$. Thus, the selection bias inherent within the Ministry's data collection process is likely to result in overestimated exclusive breast-feeding rates.

While the Ministry's coverage improves for the 3-month and 6-month Pacific estimates, there remains inherent selection bias. In 2001, the Plunket Management Information System was used to report breast-feeding at 4-6 months. However, as reported previously from a multivariable logistic model, Pacific mothers not receiving a visit from Plunket within the first six weeks postpartum had significantly lower exclusive breast-feeding rates than those who received such a visit? ${ }^{9}$ The Turnbull nonparametric survival estimates of exclusive breast-feeding rates at 6 weeks, 3 months and 6 months for those receiving a Plunket visit were 50\%, 38\% and 10\%, respectively, somewhat higher than the $45 \%, 31 \%$ and $8 \%$, respectively, of those Pacific mothers not receiving such a visit (see Table 1). Thus reliance on data solely collected from Plunket may overestimate Pacific exclusive breast-feeding rates by an absolute percentage difference of approximately $5-7 \%$ in the first three months postpartum, declining to $2 \%$ by 6 months.

Another source of difference results from the Ministry of Health's use of successive cross-sectionally derived estimates from infant age bands compared with the survival analysis using longitudinal data undertaken in our analyses. Providing follow-up rates are acceptable, as achieved in this Pacific study ${ }^{15}$, longitudinal data provide more robust estimates of time-dependent measures. Moreover, in longitudinal studies, inconsistencies in elicited responses across measurement waves and between different questions can be identified and appropriately accommodated within the analyses. For example, at the 6-week interview, 731 mothers declared that they were exclusively breastfeeding yet of these 26 were complementary feeding or fully formula-feeding at hospital discharge and a further 22 declared that they had introduced complementary liquid foods before 6 weeks. In each of these cases, mothers' breast-feeding elicitations were altered and censored in the manner described in the statistical methods. Had these responses arisen from a cross-sectional survey that elicited breast-feeding from one question, then it is likely they would have been treated as valid responses, thereby yielding overestimated rates of exclusive breast-feeding. Indeed, by treating these responses as valid observations, the proportion of mothers claiming to be exclusive breastfeeding at 6 weeks in the PIF study equalled 53\%, slightly less than the $55 \%$ reported by the Ministry ${ }^{22}$. The survival approach used in the current study also allows rate estimation to be measured at precisely defined time slices rather than broad time bands which diminish the utility of the cross-sectionally derived estimates.

A key finding in this study is the persistent difference in exclusive breast-feeding rates over infant age between Samoan and Tongan mothers, a finding first identified and reported at 6 weeks $^{12}$. This has two important implications: first, an understanding of the barriers to breastfeeding and strategies to increase breast-feeding rates should target Tongan mothers in particular ${ }^{12}$; and second, breast-feeding rates need to be monitored within the Pacific Island ethnic groups separately rather than collapsed under a 'Pacific' banner. Not only does the Pacific label serve to disguise the heterogeneity of the Pacific population in New Zealand, it is offensive to some Pacific people who value the uniqueness of their different cultures and languages as much as other ethnic groups $^{28}$. Similar ethnic differences emerged in the any breast-feeding rates at the 12-month and 24-month interviews. At these time points, the Samoan breastfeeding rates were substantially higher than the Tongan rates, with the Cook Island Maori rates being intermediary. Consistent with the literature, maternal smoking, return to employment, low birth weight and separate hospital discharge were all associated with early cessation of exclusive breast-feeding ${ }^{9,22,24-27}$. 
Another key finding presented in this paper is that inadequate education of mothers about the management of breast-feeding problems and the perceived lack of support from employers or educational facilities remain the principal reasons for mothers discontinuing exclusive breast-feeding. The same issues and recommendations for increasing exclusive breast-feeding rates that were manifest over a decade ago ${ }^{8}$ are just as pertinent today $^{1,3,13,29}$. Over half of the Pacific mothers reported introducing complementary liquid foods into their infants' diets because they were unsure about the adequacy of their milk supply, considerably higher than the 33\% recently reported from a recent New Zealand study of Caucasian women ${ }^{13}$. As concerns about insufficient breast milk supply are commonly due to mothers' poor understanding of effective techniques to increase their milk supply ${ }^{13}$, then addressing these misunderstandings during prenatal classes or visits to health-care professionals could substantially improve exclusive breast-feeding rates amongst the Pacific people.

Our presented analysis has strengths and limitations. The PIF study is a large, representative, longitudinal study with relatively small attrition rates. However, the reliance on maternal recall for the age at which complementary liquid or solid foods were introduced into infants' diets is not ideal, particularly since for half the infants these were introduced between the 6-week and 12-month interviews. In an effort to increase consistency and reliability, information gleaned about infant nutrition between and within interview surveys was cross-checked against each other. Different definitions of the exclusivity of breastfeeding can also be problematic in making comparisons between studies. The Plunket National Child Study conducted during 1990-91 defined exclusive breastfeeding as infants receiving only breast milk ${ }^{8}$ while the Ministry matched the WHO exclusive breast-feeding rate indicator ${ }^{4}$ and defined full breast-feeding as infants taking breast milk only, and no other liquids or solids except a minimal amount of water or prescribed medicines, in the past 48 hours $^{22}$. The definition of exclusive breast-feeding adopted for the PIF study was directly based on this WHO indicator, making national and international comparisons easily undertaken. Thus exclusive breast-feeding rates between the reported studies use very similar definitions. Moreover, the design of the Plunket National Child Study was longitudinal and survival analyses were also undertaken to estimate exclusive breast-feeding rates at various time points ${ }^{8}$, suggesting that the rates derived in the 1990-91 study are directly comparable to those reported for our population, after allowing for geographical differences.

Internationally, New Zealand's Pacific infant breastfeeding rates compare favourably with many of those listed in the WHO Global Data Bank ${ }^{4}$. However, in the New Zealand context, declines since the 1990s and breastfeeding rates that fall well short of WHO and other international organisations' recommendations ${ }^{1,3}$ herald the need to engage in a more concerted approach in the promotion of breast-feeding and its culture, and to dealing with the barriers or perceived barriers to breast-feeding. Mothers of Pacific infants in 2001 reported reasons for the discontinuation of breast-feeding that were largely unaltered from those cited over a decade ago. Only with the understanding of the barriers to breast-feeding within the various cultural contexts can culturally appropriate targeting, education and concerted implementation of the Ministry of Health's Action Plan for Breastfeeding effectively be undertaken. With appropriate implementation and monitoring, New Zealand may see Pacific breast-feeding rates improve and approach the recommendations of WHO and other international organisations.

\section{Acknowledgements}

The Pacific Islands Families: First Two Years of Life (PIF) study is supported by grants awarded from the Foundation for Science, Research and Technology, the Health Research Council of New Zealand, and the Maurice and Phyllis Paykel Trust. The authors gratefully acknowledge the families who participated in the study, the Pacific Peoples Advisory Board and the other members of the PIF research team. The authors also thank Dr Jane Linsday, Harvard School of Public Health, for making available the adapted IML algorithm.

\section{References}

1 American Academy of Pediatics. Breastfeeding and the use of human milk. Pediatrics 2005; 115(2): 496-506.

2 Kramer M, Kakuma R. The Optimal Duration of Exclusive Breastfeeding: A Systematic Review. Geneva: World Health Organization, 2002

3 World Health Organization (WHO). Report of the Expert Consultation on the Optimal Duration of Exclusive Breastfeeding. Geneva: WHO, 2002.

4 World Health Organization. Global Data Bank on Breastfeeding and Complementary Feeding [online], 2006. Available at http://www.who.int/nutrition/databases/infantfeeding/en. Accessed 30 May 2006.

5 Cook L, Didham R, Khawaja M. On the Demography of Pacific People in New Zealand. Wellington: Statistics New Zealand, 1999.

6 Statistics New Zealand. National Pacific Population Projections [2001(base)-2021]: Hot off the Press. Wellington: Statistics New Zealand, 2003.

7 Statistics New Zealand. Pacific Progress: A Report on the Economic Status of Pacific Peoples in New Zealand. Wellington: Statistics New Zealand, 2002.

8 Essex C, Smale P, Geddis D. Breastfeeding rates in New Zealand in the first 6 months and the reasons for stopping. New Zealand Medical Journal 1995; 108(1007): $355-7$.

9 Butler S, Williams M, Tukuitonga C, Paterson J. Factors associated with not breastfeeding exclusively among mothers of a cohort of Pacific infants in New Zealand. New Zealand Medical Journal 2004; 117(1195): U1171. 
10 Turner N, Hounsell D, Robinson E, Tai A, Whittle N. Uptake of postnatal services for mothers of newborn babies up to eight weeks of age. New Zealand Medical Journal 1999; 112(1098): 395-8.

11 Vogel A, Hutchison B, Mitchell E. Factors associated with the duration of breastfeeding. Acta Paediatrica 1999; 88(12): 1320-6.

12 Butler S, Tukuitonga C, Paterson J, Williams M. Infant feeding and feeding problems experienced by mothers of a birth cohort of Pacific infants in New Zealand. Pacific Health Dialog 2002; 9(1): 34-9.

13 Heath A-L, Tuttle C, Simons M, Cleghorn C, Parnell W. A longitudinal study of breastfeeding and weaning practices during the first year of life in Dunedin, New Zealand. Journal of the American Dietetic Association 2002; 102(7): $937-43$.

14 Statistics New Zealand. New Zealand Census of Population and Dwellings 2001: Pacific Peoples. Wellington: Statistics New Zealand, 2002.

15 Paterson J, Tukuitonga C, Abbott M, Feehan M, Silva P, Percival T, et al. Pacific Islands Families First Two Years of Life study: design and metholology. New Zealand Medical Journal 2006; 119 (1228): U1814

16 Lindsey J, Ryan L. Tutorial in biostatistics methods for interval-censored data. Statistics in Medicine 1998; 17(2): 219-38.

17 So Y. Interval-censored survival data. Paper presented at 19th Annual SAS Users Group, SAS Institute, Cary, NC, USA, 1994.

18 SAS Institute Inc. The SAS System for Windows (8.02). Cary, NC: SAS Institute Inc., 2001.
19 Statacorp. Stata Statistical Software Release 8.0. [computer package] College Station, TX: Stata Press, 2003.

20 Royal New Zealand Plunket Society. Plunket online, homepage: http://www.plunket.org.nz. Accessed 29 April 2005.

21 Sinclair R. Drop in breastfeeding rates as services cut. New Zealand Doctor 1997; 9(July): 7.

22 Ministry of Health. Breastfeeding: A Guide to Action. Wellington: Ministry of Health, 2002.

23 Rothman K, Greenland S. Modern Epidemiology, 2nd ed. Philadelphia, PA: Lippincott-Raven, 1998.

24 Howden-Chapman P, Tobias M. Social Inequalities in Health: New Zealand 1999. Wellington: Ministry of Health, 2000.

25 Clements M, Mitchell E, Wright S, Esmail A, Jones D, Ford RP. Influences on breastfeeding in southeast England. Acta Paediatrica 1997; 86(1): 51-6.

26 Ford R, Mitchell E, Scragg R, Stewart A, Taylor B, Allen E. Factors adversely associated with breast feeding in New Zealand. Journal of Paediatrics and Child Health 1994; 30(6): 483-9.

27 Galtry J. Breastfeeding and paid employment in New Zealand: what role for nurses? Nursing Praxis in New Zealand 1995; 10(3): 24-32.

28 Milne K, Kearns R. Housing status and health implications for Pacific peoples in New Zealand. Pacific Health Dialog 1999; 6 (1): 80-6.

29 World Health Organization (WHO). Global Strategy for Infant and Young Child Feeding. Geneva: WHO, 2003. 